\title{
FERTILIDADE DE CARNEIROS DE RAÇA SALOIA COM SÉMEN REFRIGERADO OU CONGELADO
}

\author{
FERTILITY OF SALOIA RAMS USING REFRIGERATED OR FROZEN SEMEN \\ Barbas, J. ${ }^{1 *}$; Marques, C. ${ }^{1}$; Baptista, M. ${ }^{1}$; Mascarenhas, R. ${ }^{1}$ Pereira, R. ${ }^{1}$; \\ Cavaco-Gonçalves, S. ${ }^{1}$; Vasques, M. ${ }^{1}$ e Horta, A. ${ }^{1}$
}

IUEBRG. L-INIA. INIAV. Vale de Santarém. Portugal. *jpbarbas@gmail.com

\section{PaLAVRAS CHAVE ADICIONAIS}

Inseminação artificial.

\section{RESUMO}

Avaliaram-se as fertilidades e coeficientes de variação $(C V)$ de carneiros Saloios após inseminação artificial (IA) por via cervical com sémen refrigerado (SR) ou congelado (SC). $\mathrm{Na} 1^{\text {a }}$ experiência avaliou-se o efeito de duas épocas de IA (primavera vs. outono) sobre a fertilidade, utilizando $S R$ ( $n=5$ carneiros) e de duas épocas de congelação de sémen (outono vs. inverno) sobre a fertilidade de ovelhas inseminadas na primavera utilizando SC ( $n=3$ carneiros). Nas outras experiências avaliaram-se as variações da fertilidade de carneiros, independentemente das épocas de IA ou congelação, utilizando SR e SC (2 $2^{\text {a }}$ experiência: 4 carneiros comuns; $3^{\text {a }}$ experiência: 16 e 5 carneiros, respectivamente). Não foram observadas diferenças na fertilidade entre épocas de IA ou congelação de sémen. As fertilidades obtidas com SR foram superiores às do SC (2 $2^{\mathrm{a}}$ experiência: 51,28 vs. $13,65 \%$; $3^{\mathrm{a}}$ experiência: 48,51 vs. $13,04 \%$ ). Os CV da fertilidade utilizando SC foram superiores aos do SR (2 ${ }^{\mathrm{a}}$ experiência: $41,3 \%$ vs. $13,1 \% ; 3^{\mathrm{a}}$ experiência: 38,9 vs. $19,5 \%$ ). Os carneiros de raça Saloia podem ser utilizados em programas de IA com SRe SC nas épocas do ano estudadas sem diminuição da fertilidade. O SC originou CV para a fertilidade superior sugerindo diferentes susceptibilidades dos carneiros à congelação.

\section{SUMMARY}

We intend to estimate Saloia breed rams fertility and its variation coefficients (VC) after cervical artificial insemination $(\mathrm{Al})$ with refrigerated $(\mathrm{SR})$ or frozen semen (SC). In experiment 1, we studied

\section{AdDITIONAL KEYWORDS \\ Artificial insemination.}

the seasonal effect of $\mathrm{Al}$ (spring vs. autumn) on fertility ( $n=5$ rams) using SR. The seasonal effect of semen freezing (autumn vs. winter, $\mathrm{n}=3$ rams) on fertility of ewes inseminated in spring was also tested with SC. Variations in ram fertility using either SF or SC were determined independently of seasons of insemination and freezing (experiment 2: SF $(n=4), S C(n=4)$, same rams; experiment 3: $S F=16, F S=5$ rams). No seasonal differences in fertility were found when ewes were inseminated either with SF or SC. Fertility was higher when RS rather than SC was used (experiment 2: $51.28 \mathrm{vs}$. $13.65 \%$; experiment 3: 48.51 vs. $13.04 \%$, respectively). Fertility VCs from SC were higher than those from SR (experiment 2: 41.3 vs. $13.1 \%$; experiment 3: 38.9 vs. $19.5 \%$, respectively). SR or SC from Saloia rams can be used in Al programs during the studied seasons with no deleterious effect on fertility. Higher fertility VCs are estimated when insemination is performed with $\mathrm{SR}$, suggesting semen individual susceptibility to freezing.

\section{INTRODUÇÃO}

A IA com SC por via cervical origina fertilidades baixas, devido às alterações que ocorrem nos espermatozóides (spz) durante o processo de congelação/descongelação, responsáveis por maiores dificuldades no trânsito espermático, diminuição da capacidade fertilizante e aumento da mortalidade embrionária (Álvarez et al., 2012). Os primeiros resultados obtidos após IA com sémen congelado (SC) originaram uma 


\section{BARBAS ETAL.}

fertilidade de $17,3 \%$ comparativamente a 40 $\%$ em animais do mesmo efectivo inseminadas com SR (Barbas et al., 2003). O objectivo deste trabalho foi avaliar as variações de fertilidade e os coeficientes de variação $(\mathrm{CV})$ em carneiros utilizados na produção de SR ou SC, independentemente das épocas de IA e de congelação do sémen. Paralelamente, comparou-se o efeito da época de IA (primavera $v s$. outono) quando utilizado SR e o efeito da época de congelação (outono vs. inverno), nas ovelhas inseminadas durante a primavera.

\section{MATERIALE MÉTODOS}

Neste trabalho foram utilizados carneiros $(n=16)$ e ovelhas multíparas $(n=200)$ da raça Saloia. O sémen foi recolhido pelo método da vagina artificial sendo avaliados o volume, as mobilidades massal (MM), individual (MI, \%), concentração $\left(10^{6} \mathrm{spz} / \mathrm{mL}\right)$, e no SC, a MI \%, e as \% de spz vivos e normais. As avaliações foram efectuadas segundo Rodriguez-Martinez (2003). Os ejaculados com uma $\mathrm{MM} \leq 3$ e $\mathrm{MI} \leq 65 \%$ foram rejeitados. O sémen destinado a ser refrigerado foi diluído com um diluidor à base de leite de vaca desnatado $(0,11 \mathrm{~g} / \mathrm{mL})$, até obter uma concentração de $1600 \times 10^{6} \mathrm{de}$ $\mathrm{spz} / \mathrm{mL}$. As palhinhas foram refrigeradas gradualmente até $15^{\circ} \mathrm{C}$. O sémen para congelar foi diluído a $30^{\circ} \mathrm{C}$ numa só etapa, utilizando um diluidor de congelação adaptado de Evans e Maxwell (1990) de modo a obter uma concentração de $1200 \times 10^{6}$ de $\mathrm{spz} / \mathrm{mL} 1$. O sémen foi refrigerado até aos $4{ }^{\circ} \mathrm{C}$ durante 4 horas. Depois, as palhinhas foram congeladas em vapores de azoto líquido durante 20 minutos. Só o SC com MI $\geq 40 \%$ e $\leq 35 \%$ de formas anormais foi utilizado na IA. No momento da retirada das esponjas vaginais foram administradas 500 UI de eCG. As IA foram realizadas por via cervical, sem prévia detecção do estro, cerca de $54 \pm 1$ horas após a remoção das esponjas com $\mathrm{SR}$, na primavera e no outono e, com SC (outono e inverno) apenas na primavera. O intervalo médio entre a recolha de sémen e a IA com SR foi de 2,5 - 4 horas. Nas IA com SC, cada ovelha foi inseminada com duas doses de sémen. A avaliação reprodutiva dos carneiros utilizados na IA foi determinada individualmente e expressa através da fertilidade. Foi também calculado o CV da fertilidade. No delineamento experimental os dados foram tratados segundo três experiências. A $1^{\mathrm{a}}$, teve como objectivo avaliar a interacção época de IA (primavera e outono) $x$ carneiro $(n=5)$ na fertilidade utilizando SR. Foram efectuadas 302 IA na primavera e 67 IA no outono, totalizando 369 IA. Na avaliação das fertilidades obtidas nas duas épocas de IA foi utilizado o teste do $\chi^{2}$, a análise de variância multivariada factorial (epoca de IA x carneiro) e o teste de Fisher. Foi avaliada a interacção época de congelação do sémen (outono e inverno) x carneiro $(n=3)$ para a fertilidade. As IA $(n=364)$ foram realizadas em maio com SC no outono $(n=144)$ e no inverno $(n=220)$. Na $2^{a}$ experiência avaliaram-se as variações da fertilidade utilizando SR ou SC. Foram utilizados 4 carneiros na produção de SR e SC que por facilidade de apresentação designaremos por carneiros comuns. Foram realizadas 172 IA com SR e 326 com SC, totalizando 498 IA. Na avaliação das fertilidades obtidas com SR e SC foi utilizada a análise de variância multivariada considerando os factores carneiro $(n=4)$ e tipo de sémen ( $S R$ e SC) (Statsoft Inc, 1995). Os CV da fertilidade de cada carneiro, foram determinados a partir do registo reprodutivo de todas as ovelhas inseminadas por cada macho e seguidamente foram comparados por análise de variância entre os grupos SR e SC $(n=4)$. $\mathrm{Na} 3^{\mathrm{a}}$ experiência avaliaram-se as variações de fertilidade usando SR ou SC de todos os carneiros. Foram realizadas 1116 IA, sendo 696 realizadas com SR e 420 com SC.

\section{RESULTADOS}

Os resultados da $1^{a}$ experiência nomeadamente as fertilidades obtidas com SR em 
duas épocas de IA, não revelaram diferenças significativas entre carneiros, épocas de IA, nem interacções significativas entre carneiros $\mathrm{x}$ épocas de IA. As fertilidades globais para os 5 carneiros nas épocas de primavera e no outono foram respectivamente $44,95 \%$ e $36,46 \%$, obtendo-se uma fertilidade média de $40,7 \%$. As fertilidades com SC no outono e no inverno foram 15,03 e $13,17 \%$. Não havendo interacções significativas carneiro $x$ época de IA $(S R)$ e carneiro x época de congelação de sémen relativamente à fertilidade ( $1^{\mathrm{a}}$ experiência $)$, este parâmetro reprodutivo foi avaliado em 4 carneiros comuns (dadores de SR e SC), independentemente da época de IA (SR) e época de congelação de sémen (SC) $\left(2^{\mathrm{a}}\right.$ experiência). Nesta experiência as fertilidades obtidas com SR $(51,28 \%)$ foram superiores às obtidas com SC $(13,65 \%)$ (tabela I). Não foram observadas variações individuais na fertilidade utilizando SR $(42,85-58,53 \%)$ ou SC (6,66-20,13\%), mas os CV para a fertilidade utilizando SC $(41,3 \%)$ foram superiores $(\mathrm{p}<0,05)$ aos obtidos com SR $(13,1$ $\%$ ) (tabela I). As fertilidades obtidas com todos os carneiros utilizados na IA com SR

Tabela I. Fertilidade de 4 carneiros comuns, dadores de sémen para refrigeração e congelação, independentemente da época de IA e da época de congelação de sémen: comparação da fertilidade entre IA com SR $e S C$. (Mean fertility of 4 common rams used for $\mathrm{Al}$ employing SR and SC independently of Al period and freezing season: fertility differences between Al with RS and SC).

\begin{tabular}{lcccccc}
\hline Sémen & $\begin{array}{c}\text { C } \\
(n)\end{array}$ & $\begin{array}{c}\text { FC } \\
(n)\end{array}$ & $\begin{array}{c}\text { DP } \\
(\%)\end{array}$ & $\begin{array}{c}\text { Min-Máx } \\
(\%)\end{array}$ & $\begin{array}{c}\text { CV } \\
(\%)\end{array}$ \\
\hline SR & 172 & 4 & $51,28^{\mathrm{a}}$ & 6,73 & $42,9-58,5$ & $13,1^{\mathrm{c}}$ \\
SC & 326 & 4 & $13,65^{\mathrm{b}}$ & 5,64 & $6,7-20,1$ & $41,3^{\mathrm{d}}$ \\
Total & 498 & & & & & \\
\hline
\end{tabular}

$\mathrm{O}=$ ovelhas $\mathrm{C}=$ carneiros $; \mathrm{FC}=$ fertilidade dos carneiros. $\mathrm{DP}=$ desvio padrão.

a vs. b; $c$ vs. d: diferenças significativas $(p<0,001)$.
Tabela II. Fertilidade de 21 carneiros independentemente da época de IA e da época de congelação de sémen: comparação entre IA com SR $(n=16)$ e $S C(n=5)$. (Mean fertility of 21 rams independently of Al period and freezing season: fertility comparison between $\mathrm{Al}$ with SR $(n=16)$ and SC $(n=5))$.

\begin{tabular}{lcccccc}
\hline Sémen & $\begin{array}{c}\text { O } \\
(n)\end{array}$ & $\begin{array}{c}\text { C } \\
(n)\end{array}$ & $\begin{array}{c}\text { FC } \\
(\%)\end{array}$ & $\begin{array}{c}\text { DP } \\
(\%)\end{array}$ & $\begin{array}{c}\text { Min-Máx } \\
(\%)\end{array}$ & $\begin{array}{c}\text { CV } \\
(\%)\end{array}$ \\
\hline SR & 696 & 16 & $48,51^{\mathrm{a}}$ & 9,45 & $28,6-63,2$ & $19,5^{\mathrm{c}}$ \\
SC & 420 & 5 & $13,04^{\mathrm{b}}$ & 5,07 & $6,7-20,1$ & $38,9^{\mathrm{d}}$ \\
Total & 1116 & 21 & & & &
\end{tabular}

$\mathrm{O}=$ ovelhas $; \mathrm{C}=$ carneiros $; \mathrm{FC}=$ fertilidade dos carneiros. $\mathrm{DP}=$ desvio padrão.

a vs. b; c vs. d: diferenças significativas $(p<0,001)$.

e com SC, independentemente das épocas de IA e das épocas de congelação de sémen ( $3^{\mathrm{a}}$ experiência), confirmam os resultados da $2^{a}$ experiência. De igual modo, as fertilidades obtidas com SR $(48,51)$ foram superiores às obtidas com SC (13,04\%) (tabela II). Não foram observadas variações individuais significativas na fertilidade utilizando SR $(28,57-63,15 \%)$ ou congelado $(6,66-20,13$ $\%)$. Os CV para a fertilidade utilizando SC $(38,9 \%)$ foram superiores aos determinados com SR $(19,5 \%)$ (tabela II).

\section{DISCUSSÃO}

Neste trabalho utilizando SR de 16 carneiros foi obtida uma fertilidade média de $48,51 \%$ em ovelhas Saloias $(n=696)$ inseminadas na primavera e no outono. Não observámos variações de fertilidade entre épocas do ano (primavera, 44,95\% vs. outono, 36,46\%) sendo possível a reprodução durante a primavera sem prejuízo da fertilidade. Não foram observadas variações significativas na fertilidade dos 16 carneiros utilizados na IA com SR, com valores compreendidos entre 28,6 e 63,2\%, registando-se um CV de 19,5\%. Não houve um efeito da época de congelação do sémen 


\section{BARBAS ETAL.}

sobre a fertilidade (outono, 15,03 \% vs. inverno, $13,17 \%$ ) nem variações individuais utilizando o SC (6,7 a 20,1\%), determinandose um CV de $38,9 \%$, que foi superior ao observado com SR $(19,5 \%)$, sugerindo diferentes aptidões à congelação/descongelação do sémen entre os carneiros de raça Saloia. As fertilidades obtidas após IA com $\mathrm{SC}$ foram influenciadas pela raça, carneiro e época de congelação do sémen. Recentemente ouve um aumento $(\mathrm{p}<0,01)$ da fertilidade (42,9 vs. 64,4\%) através da deposição vaginal de um análogo da prostaglandina E1, 6 horas antes do momento da IA com SR ( $p<0,13)$ com SC $(23,3 v s$. $32,5 \%$ ) (Barbas et al., 2013). A IA com SC originou uma diminuição significativa da fertilidade sendo o decréscimo de fertilidade atribuído a vários factores nomeadamente a duração da capacidade fertilizante (Morrier

\section{BIBLIOGRAFIA}

Álvarez, M.; Chamorro, C.; Kaabi, M.; Anel-Lopez, L.;Boixo, J.;Anel, E.; Anel, L. and De Paz, P. 2012. Design and in vivo evaluation of two adapted catheters for intrauterine transcervical insemination in sheep. Anim Reprod Sci, 131: 153-159.

Barbas, J.; Horta, A.; Baptista, M. e Marques, C. 2003. Variação na capacidade fertilizante do sémen refrigerado e congelado em ovelhas de raça Saloia. IV Congresso Ibérico de Reproduccion Animal. Federación lbérica de Reproducción Animal. Arucas. Las Palmas. Espanha. Ponencias y Comunicaciones, 63 (Resumo).

Barbas, J.; Horta, A.; Marques, C.; Baptista, M.; Mascarenhas, R.; Martins, D.; Vasques, M.; Pereira, R. and Cavaco-Gonçalves, S. 2013. The fertility increase after misoprostol administration is differently expressed when sheep are inseminated with chilled or frozen-thawed sémen. Small Ruminant Res, http://dx.doi.org/ e Bailey, 2012), a proporção de spz móveis e respectiva aptidão para ultrapassar o cérvix ovino (Richardson et al., 2012).

\section{CONCLUSÕES}

Os carneiros de raça Saloia podem ser utilizados em programas de IA com SR ou SC nas épocas do ano estudadas sem decréscimo da fertilidade. A congelação do sémen dos carneiros da raça Saloia origina uma diminuição significativa da fertilidade determinado-se um aumento significativo do respectivo $\mathrm{CV}$. Não foram observadas variações individuais significativas na qualidade do SC e na fertilidade dos carneiros após IA com SC. O elevado CV da fertilidade utilizando SC sugere diferentes susceptibilidades entre carneiros na aptidão à congelação/descongelação dos respectivos ejaculados.

10.1016/j.smallrumres.2013.04.011.

Evans, G. y Maxwell, W. 1990. Inseminación artificial de ovejas y cabras. Zaragoza. España. Editorial Acribia, S.A. (Ed.): 1-192.

Rodriguez-Martinez, H. 2003. Laboratory semen assessment and prediction of fertility: still utopia?. Reprod Domest Anim, 38: 312-318.

Morrier, A. and Bailey, J. 2012. Pre-incubation prior to semen processing and the subsequent effect on the quality of fresh-cooled and cryopreserved ram semen. Small Ruminant Res, 102: 57-62.

Richardson, L.; Hanrahan, J.; Donovan, A.; Marti, J.; Fair, S.; Evans, A. and Lonergan, P. 2012. Effect of site of semen deposition on the fertility of sheep inseminated with frozen-thawed semen. Anim Reprod Sci, 131: 160-164.

Statsoft Inc. 1995. Statistica for Windows [Computer program manual]. Tulsa. OK. 